\title{
ACCIDENTALIDAD VIAL POR CONSUMO DE DROGAS Y ALCOHOL: ESTADO ACTUAL
}

Camilo Uribe Granja MD, MSc*, Miguel Ángel Saavedra Ortiz MD**, José Rafael Almarales Navarro MD***, Lorenzo Eduardo Cabezas Pulido MD***, Pedro Vicente Soto Bohórquez MD***

\section{Resumen}

Introducción: el consumo de alcohol se reconoce como causa importante de accidentes de tránsito por el efecto depresor sobre el sistema nervioso central. Aunque las drogas recreacionales o ilegales tienen una acción similar es poco lo que se conoce sobre su relación con la accidentalidad vial. Objetivo: conocer la situación actual en Colombia y el mundo sobre los efectos del alcohol y las sustancias psicoactivas ilegales en el sistema nervioso central como posibles causas de accidentes de tránsito. Métodos: revisión de la literatura en bases electrónicas utilizando los términos DeCS y MeSH: street drugs, designed drugs, ethanol, cocaine, cannabinoides, amphetamines, injuries, accident traffic y automobile driving. Resultados: algunos estudios en especial en Estados Unidos, se reporta una relación estrecha entre el consumo de sustancias psicoactivas y la accidentalidad vial, con cifras cercanas a las del alcohol. En México también se ha demostrado esta relación. En Colombia la mayoría de publicaciones sobre factores de accidentalidad vial no diferencian el consumo de alcohol de las sustancias psicoactivas, lo cual refleja la falta de programas de promoción y prevención sobre el riesgo de conducir bajo la acción de estas sustancias. Los exámenes para determinar su presencia no se ordenan en forma rutinaria y por los datos existentes no reflejan los costos reales derivados de la atención a víctimas de accidentes de tránsito causados por conducir bajo sus efectos. Conclusiones: la situación actual sobre la accidentalidad vial muestra un preocupante aumento de la mortalidad, morbilidad y discapacidad en países en vías de desarrollo como el nuestro. Se analizaron los efectos del alcohol, cocaína, canabinoides y metanfetaminas sobre el sistema nervioso central que disminuyen las habilidades del individuo para la conducción.

Palabras clave: drogas ilícitas, drogas diseñadas, etanol, accidentes de tránsito, lesiones.

Abreviaturas: SNC, sistema nervioso central; AT, accidentes de tránsito.

\section{ROAD TRAFFIC ACCIDENTS RELATED TO DRUGS AND ALCOHOL: CURRENT SITUATION}

\section{Abstract:}

Introduction: alcohol consumption is acknowledged as an important cause of road accidents due to its depressing effect on the central nervous system. Although recreational or illegal drugs have a similar action little is known on their relation with road accidents. Objective: to determine the current situation in Colombia and worldwide on the effects of alcohol and illegal psychoactive substances on the central nervous system as possible causes of road accidents. Methods: a review of the literature following an online data base search using DeCS and MeSH

Fecha recibido: diciembre 14 de 201 I - Fecha aceptado: febrero 29 de 2012

* Director científico, Fundación Unidad Integral de Toxicología (UNITOX) y coordinador de Toxicología Clínica, Hospital Infantil Universitario de San José. Profesor Asociado, Fundación Universitaria de Ciencias de la Salud, Bogotá DC. Colombia.

\footnotetext{
** Jefe de Posgrado de Medicina de Urgencias, Profesor Asociado, Fundación Universitaria de Ciencias de la Salud. Jefe del Servicio de Urgencias, Hospital de San José. Bogotá DC. Colombia.

*** Médico residente de Medicina de Urgencias, Facultad de Medicina, Fundación Universitaria de Ciencias de la Salud. Bogotá DC. Colombia.
} 
terms: street drugs, designed drugs, ethanol, cocaine, cannabinoides, amphetamines, injuries, traffic accident and automobile driving. Results: particularly in the United States some trials report a close relationship between the use of psychoactive substances and road accidents rendering figures close to those of alcohol. This relation has also been demonstrated in Mexico. Most of the publications on road accident factors in Colombia do not differentiate alcohol consumption from psychoactive substances use. This reflects a lack of promotion and prevention programs on alcohol and substance-related risk when driving. Tests to determine their presence are not ordered in a routine manner and available data does not reflect the actual costs related to care provided to victims of accidents caused by driving under the influence of these substances. Conclusions: the current situation of road accidents shows a distressing increase in mortality, morbidity and disability rates in developing countries such as our country. The effects of alcohol, cocaine, cannabinoides and methamphetamines on the central nervous system were analyzed concluding they affect a person's ability to drive safely.

Key words: illegal drugs, designed drugs, ethanol, car accidents, injuries.

\section{Introducción}

\section{Situación mundial}

Los AT son un problema de salud pública con una alta carga social para el individuo y su familia, asícomo para la economía de los países. ${ }^{1}$ Según las estadísticas de la Organización Mundial de la Salud (OMS) y el Banco Mundial para $2009^{2}$, cada año mueren en el mundo 1,2 millones de personas por causa de AT en la vía pública y cerca de 50 millones resultan heridas. ${ }^{3}$ Las proyecciones indican que a menos que haya un renovado compromiso con la prevención, estas cifras aumentarán cerca del $65 \%$ durante los próximos 20 años. ${ }^{1}$

\section{Etanol}

Es bien conocido que el consumo de alcohol aumenta la probabilidad de choques causantes de muerte o de traumatismos graves, debido a su efecto depresor sobre el SNC. ${ }^{4}$ La ingestión de cantidades moderadas de etanol puede tener efectos antiansiedad y acciones que producen desinhibición del comportamiento en una amplia gama de dosis. ${ }^{5-7}$ Los signos individuales de intoxicación varían desde un ánimo expansivo y vivaz, a cambios de humor descontrolados y arrebatos emocionales que pueden tener componentes violentos. En la intoxicación severa por lo general la función del SNC se ve afectada y se produce una condición de anestesia general, que prevalece en última instancia en relación con cambios específicos en neurotransmisores. ${ }^{8-11}$ Sin embargo, hay poco margen entre los efectos anestésicos y los letales, casi siempre debido a la depresión respiratoria. ${ }^{12}$ Grandes dosis de etanol pueden interferir con la fijación de los recuerdos, produciendo amnesias anterógradas, conocidas como apagones o lagunas alcohólicas, por lo que las personas afectadas son incapaces de recordar todo o parte de las experiencias durante el período de la ingesta de alta cantidad. ${ }^{12 .}{ }^{13}$ Incluso, se considera al alcohol como causa importante de demencia ${ }^{14}$ debido a que el abuso produce contracción del cerebro debido a la pérdida de materias blanca y gris ${ }^{15-17}$, así como disminución del metabolismo cerebral de la glucosa ${ }^{18}$ y pérdida de la función neuronal ${ }^{19}$, dependiendo en ambos casos de la cantidad y la duración del consumo. ${ }^{20}$

En la ingestión aguda la sintomatología está relacionada en forma directa con la concentración de alcohol en la sangre y por ende con la impregnación de la misma sustancia en el SNC. ${ }^{21}$ Con cifras sanguíneas hasta $20 \mathrm{mg} \%$ no existe ninguna alteración; entre 20 y $50 \mathrm{mg} \%$ puede haber alguna locuacidad y merma de reflejos; de 50 a $85 \mathrm{mg} \%$ hay disminución de los reflejos y alteración en la percepción y entre 85 y 100 mg \% en una tercera parte de las personas puede haber síntomas de embriaguez y las inhibiciones sociales están disminuidas, las respuestas se tornan lentas y ya existe incoordinación. A niveles de 100 a 150 mg \% la mitad están ebrias y hay definida disminución en la capacidad de respuesta y de la actividad motora. ${ }^{21}$ 
Estos datos se refieren al comportamiento en actividades sociales, porque en actuaciones que exigen precisión como lo es la conducción de un vehículo automotor, cifras superiores a $100 \mathrm{mg} \%$ llevan a alteraciones psicomotrices para el manejo de vehículos. Con cifras de 150 a $200 \mathrm{mg} \%$, el 80\% está ebrio y existe percepción defectuosa en sentidos tan importantes como la visión, disminución al dolor y la voz es mal articulada. De $200 \mathrm{mg} \%$ en adelante cualquiera estará completamente ebrio. De 250 a 300 mg \% existe disminución a los estímulos, notoria incoordinación muscular que torna difícil mantenerse en pie. Valores de $300 \mathrm{mg} \%$ en adelante hacen que el individuo esté en estupor y variará de superficial a profundo. Más de $400 \mathrm{mg} \%$ llevan a coma, hipotermia, hiporreflexia, anestesia y colapso, que con frecuencia son fatales. De $500 \mathrm{mg} \%$ en adelante sobreviene depresión de los centros respiratorio y vasomotor y fallecen. Entre 600 y $700 \mathrm{mg} \%$ hay coma profundo con muerte rápida. Alcoholemias por encima de $700 \mathrm{mg} \%$ son incompatibles con la vida. ${ }^{21}$

Sin embargo, la embriaguez aguda no solo puede estar causada por el consumo de etanol sino también por otras sustancias psicoactivas como cocaína, marihuana y demás psicofármacos. ${ }^{22}$ Se tienen indicios de que entre los conductores ha aumentado su ingesta tanto con fines medicamentosos como recreacionales, a menudo en combinación con alcohol ${ }^{23}$, pero sus efectos sobre la conducción y la inducción de AT han sido menos estudiados que los del alcohol.

\section{Cocaína}

En el caso de la cocaína, su consumo en combinación con el etanol forma el compuesto cocaetileno que aumenta tanto la duración como la intensidad de los efectos llevando a mayor euforia, hiperactividad, conductas de riesgo ${ }^{24}$, taquiarritmias, paro cardíaco, eventos coronarios y convulsiones. ${ }^{25}$ Como droga de abuso se ha visto asociada con la realización de actos violentos. ${ }^{26}$ Cualquiera que sea su procesamiento o vía de administración que da como productos finales base de coca libre, crack o bazuco ${ }^{27}$, se absorbe en forma rápida dando lugar a una serie de efectos simpaticomiméticos, generando cambios como taquicardia, hipertensión arterial, midriasis y broncoespasmo. ${ }^{28} \mathrm{~A}$ nivel del SNC la cocaína aumenta la permeabilidad de la barrera hematoencefálica favoreciendo aún más su entrada. ${ }^{29}$

El principal efecto de la cocaína se centra en el incremento en la liberación de la dopamina en las uniones sinápticas y bloqueo de los transportadores presinápticos impidiendo su recaptación. ${ }^{30} \mathrm{~A}$ mayor concentración de dopamina hay más euforia. ${ }^{31}$ La cocaína causa también inhibición de la recaptación de los neurotransmisores noradrenalina y serotonina, que son los principales responsables de los cambios asociados con conductas imprudentes que pueden llevar a AT con lesiones subsecuentes. ${ }^{24}$

\section{Canabinoides}

Otra de las drogas ilícitas de mayor abuso es la marihuana. Su uso es conocido desde hace más de 4.000 años para uso medicinal en China e India ${ }^{32}$ y en la actualidad se usa con fines médicos para el manejo del vómito incoercible en quimioterapia y como estimulante del apetito en pacientes VIH positivos. ${ }^{32} \mathrm{Hoy}$ prevalece su consumo en países del primer mundo y afecta sobre todo a los grupos poblacionales del género masculino y adolescentes. ${ }^{33}$

Los efectos del consumo de marihuana en el organismo se deben a la acción de los canabinoides en los receptores específicos CB1 y CB2 ubicados en especial en el SNC y células linfocitarias. ${ }^{32}$ Se presentan efectos adversos como disfunción eréctil, supresión de hormonas sexuales, complicaciones pulmonares y de la modulación del sistema inmune celular y humoral. ${ }^{32}$ En el SNC inhibe la liberación de norepinefrina, dopamina, glutamato y ácido aminobutírico en las uniones sinápticas del globus pallidus, sustancia nigra, cerebelo, hipocampo y corteza cerebral. ${ }^{32,34} \mathrm{El}$ consumo de marihuana produce alteraciones de la percepción del individuo y del entorno, letargia, ortostatismo, imposibilidad para concentrarse, episodios de pánico y psicosis aguda, limitando al paciente para desarrollar actividades que impliquen coordinación motora y por lo tanto, su habilidad para maniobrar un vehículo automotor. ${ }^{35}$ Inclusive, su consumo crónico puede 
desatar esquizofrenia en aquellos con predisposición genética. ${ }^{35,36}$

Dados sus efectos sobre el SNC, se ha buscado identificar el consumo de canabinoides como factor de riesgo para conducir, encontrando una fuerte asociación con el uso combinado de etanol ${ }^{37,38}$ y así mismo aumento de conductas de riesgo en relación con la psicosis aguda, hallando canabinoides positivos hasta en $6 \%$ del total de víctimas de homicidio en Estados Unidos ${ }^{39} \mathrm{y}$ en forma paradójica un $14 \%$ en un estudio llevado a cabo en Colombia. ${ }^{26}$

\section{Anfetaminas}

Las anfetaminas fueron sintetizados por primera vez en 1914 y se utilizaron al principio para control del asma, ${ }^{27}$ como inhibidores del apetito y para el manejo de narcolepsia. Sin embargo, muy pronto se cayó en el abuso y muy rápido se prohibió por la FDA (Food and Drug Administration) en los años sesenta. ${ }^{40}$ Desde su descubrimiento, la anfetamina original (fenilisopropilamina) ha sufrido variaciones con el fin de obtener mayores efectos para su uso recreativo. De su molécula original se han creado más de 200 derivados, siendo el más usado hoy en día el MDMA (D-fenilisopropilmetilamina hidroclorato), también conocido como éxtasis. ${ }^{27}$

Las anfetaminas se absorben muy rápido por vía oral y se metabolizan en el hígado. Su mecanismo de acción se basa en la liberación de catecolaminas, sobre todo dopamina y norepinefrina, en las uniones sinápticas. ${ }^{31}$ La dopamina una vez producida se acumula en vacuolas acidófilas que se almacenan en la unión presináptica ${ }^{31}$ que son lisadas por el efecto de las anfetaminas, además de generarse un aumento en su producción por catalización de la vía enzimática para tal fin. ${ }^{31}$ Sin embargo, esta sobreproducción de dopamina y norepinefrina con aumento de su concentración presináptica lleva a degeneración neuronal y daño citoplasmático. ${ }^{41,42}$ Ante el aumento de los niveles de catecolaminas sobreviene la euforia, agitación, conductas violentas y de riesgo con duración variable de cuatro a seis horas, ${ }^{27}$ que contraindican su consumo con la conducción.

\section{Situación en Colombia}

En Colombia el Fondo de Prevención Vial y el Instituto Nacional de Ciencias Forenses y Medicina Legal junto con diversas instituciones, vienen recopilando cifras sobre la accidentalidad a nivel nacional en las vías rurales de las capitales y los departamentos desde 1997. ${ }^{43,44}$ Esto ha conducido a reconocer los accidentes por conducción bajo efectos de etanol como la causa aislada más importante de muertes relacionadas con el consumo de alcohol ${ }^{45}$ y por lo tanto han conducido a implementar programas para la promoción, prevención, concientización y educación de la ciudadanía acerca del riesgo de conducir bajo este efecto con resultados muy exitosos..$^{22,45-47}$ Sin embargo, como no existen datos semejantes por el consumo de sustancias psicoactivas, tampoco hay hasta el momento protocolos para registrar su presencia en las víctimas de AT y así poder determinar su relación con los diferentes tipos de accidentes y gravedad del trauma, para implementar políticas de seguridad vial semejantes a las que existen con el alcohol.

Dados los efectos del etanol y las sustancias psicoactivas sobre el SNC y sus implicaciones en la coordinación y los reflejos, es importante hacer una revisión de la literatura médica científica sobre su consumo en víctimas de AT con el fin de identificar sus fortalezas, debilidades y alcances, para así plantear una metodología adecuada que permita realizar un estudio preliminar en Bogotá.

\section{Métodos}

Se realizó una búsqueda sistemática de la literatura en bases de datos especializadas, entre 1990 y 2011. Se consultaron datos: PUBMEDLINE (National Library of Medicine Database), HINARI, SciELO y EMBASE, utilizando los términos DeCS y MeSH: street drugs, designed drugs, ethanol, cocaine, cannabinoides, amphetamines, injuries, accident traffic y automobile driving.

Se usó el programa Reference Manager 10 para organizar las referencias bibliográficas, eliminar 
duplicados ỳ realizar las respectivas bibliografías. Los títulos o resúmenes recuperados fueron revisados para seleccionar los artículos teniendo en cuenta la relevancia y aplicabilidad en nuestro contexto sociodemográfico.

\section{Resultados}

La revisión realizada de la literatura coincide con las graves implicaciones que la accidentalidad tiene en cuanto a mortalidad, discapacidad y costos, y cómo estas pueden variar en forma positiva o negativa con el tiempo dependiendo del desarrollo de los países y la implementación de medidas de prevención. ${ }^{48,49}$ Así mismo, se ha detectado que el consumo de etanol está implicado en las víctimas de AT ya sea que se trate de conductores, peatones o motociclistas, y que este impacta en la mortalidad y discapacidad de los individuos en los países en vías de desarrollo. ${ }^{48,50}$

En Estados Unidos se ha estudiado a fondo la asociación entre embriaguez alcohólica, traumatismos por accidentes de tránsito y discapacidad asociada con la dependencia del alcohol. ${ }^{51}$ Incluso se conocen cifras oficiales sobre el costo económico de los AT asociados con el consumo de alcohol (13,619 millones de dólares americanos), más no del asociado con el consumo de drogas. ${ }^{52}$ En Canadá también se tienen datos sobre los costos impuestos sobre los sistemas de salud por el consumo de alcohol y drogas ilegales. ${ }^{53}$

En Estados Unidos se han realizado varios estudios que examinan el consumo de drogas ilícitas en los conductores involucrados en colisiones vehiculares, conducción imprudente o accidentes fatales. Incluso hay algunos que muestran cómo la presencia de "solo drogas" en las víctimas de AT es casi el doble que la 87 del consumo de "solo alcohol" y casi el triple que drogas y alcohol. ${ }^{54}$

Las cifras indican que el $18 \%$ de los conductores heridos de gravedad en AT durante 2009 resultaron positivos para el consumo de drogas ilícitas, fármacos prescritos o de venta libre, mientras que la misma cifra para 2005 era de $13 \% .55$
En la Unión Europea el abuso en el consumo de cannabis, ya sea solo o en combinación con alcohol, es causa de gran preocupación. De hecho, tres millones de europeos consumen a diario cannabis y el $80 \%$ reporta conducir luego de hacerlo. ${ }^{56}$ Estudios realizados entre 1982 y 2003 en varios países desarrollados, entre estos Canadá ${ }^{57,58}$, Estados Unidos ${ }^{59-61}$ Alemania $^{62,63}$ y Australia ${ }^{64,65}$ revelan que entre $4 \mathrm{y}$ $14 \%$ de los conductores que sufrieron heridas o murieron como consecuencia de un AT tenían niveles en sangre de delta-9-tetrahidrocanabinol (THC), el ingrediente activo de la marihuana, de los cuales entre 50 y $80 \%$ también había ingerido alcohol. ${ }^{56}$ Estudios de casos y controles concuerdan con los análisis experimentales que demuestran que el THC afecta las funciones cognitivas, psicomotoras y la conducción de vehículos motorizados. ${ }^{61}$ Existe poca evidencia sobre si el consumo de cannabis por si solo aumenta la probabilidad de sufrir un AT; sin embargo, se ha determinado que la medición de niveles sanguíneos demuestra como las cifras altas de THC cursaron con una probabilidad tres o cuatro veces mayor de causar AT, en comparación con aquellos que no han utilizado alcohol o drogas. Así mismo se ha observado que ante niveles de THC por encima de $30 \mu \mathrm{g} / \mathrm{k}$ se producen efectos sobre el desempeño cognitivo, psicomotor y la habilidad para manejar, semejantes a los causados por niveles de alcohol en sangre $\geq 0.05$ $\mathrm{g} / \mathrm{dl}$, que corresponden al límite legal para conducir en la mayoría de países europeos ${ }^{66}$ Estudios de corte transversal realizados en África muestran una asociación significativa entre consumo de alcohol y uso de estimulantes del SNC (marihuana y nicotina) con la ocurrencia de AT. ${ }^{67}$

En España es frecuente detectar sustancias ilegales en víctimas de AT. Un estudio realizado el año 2000 reporta su presencia en $10,2 \%$ de los pacientes lesionados por esta causa. Se detectó una sola sustancia en $2,5 \%$ de las muestras y en combinación con otras sustancias en 7,7\%, siendo la cocaína la más común. En el $68,9 \%$ de los casos en que se identificó una sustancia ilegal, se encontraba en niveles tóxicos. ${ }^{68}$

En América Latina son pocos los datos que se han obtenido con respecto al uso de sustancias ilícitas. En 
un estudio prospectivo de corte transversal realizado en Brasil se determinó la prevalencia del consumo de sustancias psicoactivas en personas admitidas al servicio de urgencias seis horas después de sufrir una lesión no fatal. Los resultados mostraron que $11 \%$ tenía niveles positivos de alcohol y $10 \%$ algún grado de intoxicación. De 242 pacientes, 13,6\% resultaron positivos para cannabis, 3,3\% para cocaína y $4,2 \%$ para benzodiacepinas. ${ }^{69}$

En Colombia las cifras del DANE (Departamento Administrativo Nacional de Estadística) indican que los AT son hoy la quinta causa de muerte. Fueron responsables de 3.722 AVISAS (años de vida saludables perdidos) por mortalidad y 0,064 por discapacidad..$^{7 \bullet}$ Esto significa que los AT en Colombia tienen una alta letalidad y un gran número de víctimas sobreviven padeciendo discapacidad.

Según las cifras publicadas por el Instituto Nacional de Medicina Legal y Ciencias Forenses, la embriaguez se encuentra dentro de las primeras causas de accidentalidad vial. Sin embargo, dicho instituto reporta que cerca del $23 \%$ de los AT cuya causa fue identificada, se debieron a exceso de velocidad y solo el $8 \%$ se atribuyeron a embriaguez, sin que exista una diferenciación entre la de tipo alcohólica o por otro tipo de sustancias. ${ }^{71}$ Es posible que las drogas ilícitas se encuentren relacionadas con un porcentaje significativo de la cifra de accidentes por exceso de velocidad, dada la relación de su consumo con comportamientos de riesgo.

\section{Discusión}

En general los estudios muestran que el consumo de sustancias psicoactivas ilegales es cada vez mayor en países industrializados, así como en aquellos en vías de desarrollo y por lo tanto su impacto debe tenerse en cuenta en los programas de prevención de AT. Entre las drogas ilícitas con mayor consumo se encuentran la cocaína y las metanfetaminas, debido a su efecto estimulatorio sobre la sobreproducción de catecolaminas con la correspondiente inducción de trastornos de hiperactividad, así como de conductas impulsivas y de riesgo. De la misma manera se ha identificado que el consumo de canabinoides genera alteración de la percepción propia y del entorno, lo cual disminuye la capacidad de reacción, conduce a psicosis y se reconoce que su consumo combinado con etanol aumenta el riesgo de accidentalidad vial.

La revisión de la literatura destaca que Colombia carece de evidencia fuerte, ya que no existen estudios que asocien los accidentes de tránsito con el consumo de estas sustancias, como si los hay en algunos países. Por ello no se registran datos precisos sobre la carga económica que generan los traumatismos derivados del tráfico en este grupo de pacientes, de tal manera que las políticas actuales no han contemplado el consumo de sustancias psicoactivas en los controles de seguridad vial.

Como existe un vacío en el conocimiento, sería conveniente realizar estudios que determinen la prevalencia del consumo de sustancias psicoactivas en los pacientes víctimas de AT que ingresan a los hospitales, para así determinar el impacto real sobre los sistemas de salud y evaluar si el aporte transferido por las industrias licoreras a los departamentos por concepto de impuesto a la venta de bebidas alcohólicas es el adecuado.

Se propone realizar un estudio en un servicio de urgencias de IV nivel en el que se atiendan un número importante de pacientes víctimas de AT (ya sea conductor, pasajero o peatón) con diagnóstico de politraumatismo moderado o severo, en el que se determinen niveles de alcoholemia y sustancias psicoactivas como cocaína, metanfetaminas, marihuana, antidepresivos tricíclicos, opiáceos y benzodiacepinas en aquellos pacientes con alteración de conciencia determinado por la escala de coma de GLASGOW (puntaje menor a 12/15) y diagnóstico de traumatismo moderado a letal según puntaje de ISS (injury severity score).

Teniendo en cuenta las variables sociodemográficas de la población a estudio, es importante describir la relación entre la severidad del trauma, el consumo de sustancias psicoactivas y su distribución según la edad, así como establecer la frecuencia del trauma de acuerdo con la zona corporal afectada en pacientes bajo 
efectos de alcohol y sustancias psicoactivas, y conocer el costo total de la atención en salud de las víctimas de AT bajo los efectos del alcohol y sustancias psicoactivas. Conviene determinar la concordancia entre la prueba de embriaguez clínica y el resultado de alcohol en sangre, para lo cual es fundamental capacitar a los médicos de urgencias para que se basen en el reglamento técnico forense para establecer el estado de embriaguez del Instituto Nacional de Medicina Legal y Ciencias Forenses bajo la supervisión del médico toxicólogo, ya que una mala interpretación del médico evaluador induce a sesgos en los resultados.

Un estudio de dichas características permitiría implementar un protocolo para la búsqueda de alcohol y otras sustancias psicoactivas en pacientes politraumatizados víctimas de AT que ingresan a los servicios de urgencias en cualquier hospital de Colombia. Además, aportaría datos para proponer un proyecto de ley que endurezca las penas por transitar en estado de embriaguez alcohólica, tanto en conductores como en peatones. Este proyecto serviría de insumo para acelerar el proceso de reglamentación de la enmienda constitucional, Acto Legislativo 01 de 2009, sobre el consumo de sustancias psicoactivas ilegales.

\section{Referencias}

1 Peden M., editor. Informe mundial sobre prevención de los traumatismos causados por el transito[monografía en Internet].Ginebra: OMS; 2004 [citado 23 Mar 2012]. Disponible en: http://www.paho.org/spanish/dd/pub/resumen_informe_mundial_traumatismos.pdf

2 Clasificación de países [monografía en Internet].Washington: Banco Mundial 2010. [citado 23 Mar 2012]. Disponible en: http://datos.bancomundial.org/quienes-somos/clasificacion-paises

3 Informe sobre la situacion mundial de la seguridad vial: es hora de pasar a la accion. Ginebra : OMS; 2009.

4 Sakurai S, Cui R, Tanigawa T, Yamagishi K, Iso H. Alcohol consumption before sleep is associated with severity of sleep-disordered breathing among professional Japanese truck drivers. Alcohol ClinExp Res. 2007 Dec;31(12):2053-8.

5 Pastor R, Aragon CM. The role of opioid receptor subtypes in the development of behavioral sensitization to ethanol. Neuropsychopharmacology. 2006 Jul;31(7): 1489-99.

6 Job MO, Tang A, Hall FS, Sora I, Uhl GR, Bergeson SE, et al. Mu (mu) opioid receptor regulation of ethanol-induced dopamine response in the ventral striatum: evidence of genotype specific sexual dimorphic epistasis. Biol Psychiatry 2007 Sep 15;62(6):627-34

7 Voronin K, Randall P, Myrick H, Anton R. Aripiprazole effects on alcohol consumption and subjective reports in a clinical laboratory paradigm--possible influence of self-control. Alcohol ClinExp Res. 2008 Nov;32(11): 1954-61.

(8) Aryal P, Dvir H, Choe S, Slesinger PA. A discrete alcohol pocket involved in GIRK channel activation. Nat Neurosci. 2009 Aug;12(8):988-95.
9 Davies AG, Pierce-Shimomura JT, Kim H, VanHoven MK, Thiele TR, Bonci A, et al. A central role of the BK potassium channel in behavioral responses to ethanol in C. elegans.Cell. 2003 Dec 12;115(6):655-66.

10 Dopico AM, Chu B, Lemos JR, Treistman SN. Alcohol modulation of calciumactivated potassium channels. Neurochem Int. 1999 Aug;35(2):103-6.

11 Steensland P, Simms JA, Holgate J, Richards JK, Bartlett SE. Varenicline, an alpha4beta2 nicotinic acetylcholine receptor partial agonist, selectively decreases ethanol consumption and seeking. ProcNatlAcadSci U S A. 2007 Jul 24;104(30):12518-23.

12 Schuckit M. Ethanol and Methanol. In: Brunton L, editor. Goodman \& Gilman's The Pharmacological Basis of Therapeutics. 12th ed. New York: McGraw-Hill; 2010. p. 629-48.

13 Stephens R, Ling J, Heffernan TM, Heather N, Jones K. A review of the literature on the cognitive effects of alcohol hangover. Alcohol Alcohol 2008 $\operatorname{Mar} ; 43(2): 163-70$.

14 Oslin D, Atkinson RM, Smith DM, Hendrie H. Alcohol related dementia: proposed clinical criteria. Int J Geriatr Psychiatry. 1998 Apr;13(4):203-12.

15 Pfefferbaum A, Sullivan EV, Rosenbloom MJ, Mathalon DH, Lim KO. A controlled study of cortical gray matter and ventricular changes in alcoholic men over a 5-year interval.Arch Gen Psychiatry. 1998 Oct;55(10):905-12.

16 Kril JJ, Halliday GM. Brain shrinkage in alcoholics: a decade on and what have we learned?.ProgNeurobiol. 1999 Jul;58(4):381-7.

17 Alexander-Kaufman K, Harper C, Wilce P, Matsumoto I. Cerebellar vermis proteome of chronic alcoholic individuals. Alcohol ClinExp Res. 2007 Aug;31(8): 1286-96.

18 Volkow ND, Wang GJ, Hitzemann R, Fowler JS, Overall JE, Burr G, et al. Recovery of brain glucose metabolism in detoxifiedalcoholics. Am J Psychiatry. 1994 Feb;151(2):178-83.

19 Baker KG, Harding AJ, Halliday GM, Kril JJ, Harper CG. Neuronal loss in functional zones of the cerebellum of chronic alcoholics with and without Wernicke's encephalopathy. Neuroscience 1999;91(2):429-38.

20 Bartsch AJ, Homola G, Biller A, Smith SM, Weijers HG, Wiesbeck GA, et al Manifestations of early brain recovery associated with abstinence from alcoholism. Brain. 2007 Jan;130(Pt 1):36-47.

21 Yip L. Ethanol. In: Nelson L, Lewin N, Howland MA, Hoffman R, Goldfrank L, Flomenbaum N, editors. Goldfrank'sToxicologic Emergencies. 9thed. New York: McGraw Hill; 2011.

22 OPS. Beber y Conducir: manual de seguridad vial para decisores y profesionales. Washington: OPS; 2010.

23 OPS. Defunciones por accidentes de transito en paises seleccionados de las americas 1985-2001. Bol. Epidemiol. 2004 Mar;25(1):2-5.

24 Filip M, Alenina N, Bader M, Przegalinski E. Behavioral evidence for the significance of serotoninergic (5-HT) receptors in cocaine addiction. Addict Biol. 2010 Jul;15(3):227-49.

25 Phillips K, Luk A, Soor GS, Abraham JR, Leong S, Butany J. Cocaine cardiotoxicity: a review of the pathophysiology, pathology, and treatment options. Am J CardiovascDrugs. 2009;9(3):177-96.

26 Bravo P, Bravo S. Prevalencia de sustancias psicoactivas asociadas con muertes violentas en Cali. ColombMed. 2005 Jul 1;36(3):146-52.

27 Demaria S Jr, Weinkauf JL. Cocaine and the club drugs. IntAnesthesiolClin. 2011;49(1):79-101.

28 Goldstein RA, DesLauriers C, Burda AM. Cocaine: history, social implications, and toxicity--a review. Dis Mon 2009 Jan;55(1):6-38.

29 Dietrich JB. Alteration of blood-brain barrier function by methamphetamine and cocaine. Cell Tissue Res. 2009 Jun;336(3):385-92.

30 Prosser JM, Hoffman R. Cocaine. In: Nelson L, Lewin N, Howland MA, Hoffman R, Goldfrank L, Flomenbaum N, editors. Goldfrank's Toxicologic Emergencies. 9thed. New York: McGraw Hill; 2010.

31 Seger D. Cocaine, metamfetamine, and MDMA abuse: the role and clinical importance of neuroadaptation. ClinToxicol (Phila). 2010 Aug;48(7):695-708. 
McGuigan M. Cannabinoids. In: Nelson L, Lewin N, Howland MA, Hoffman R, Goldfrank L, Flomenbaum N, editors. Goldfrank'sToxicologic Emergencies. 9thed. New York: McGraw Hill; 2010.

33 Copeland J, Swift W. Cannabis use disorder: epidemiology and management. Int Rev Psychiatry. 2009 Apr;21(2):96-103.

34 Koob G, Kreek MJ. Stress, dysregulation of drug reward pathways, and the transition to drug dependence. Am J Psychiatry. 2007 Aug; 164(8):I 149-59.

DeLisi LE. The effect of cannabis on the brain: can it cause brain anomalie that lead to increased risk for schizophrenia?.CurrOpin Psychiatry. 2008 Mar;21(2): 140-50.

36 Schuckit MA. Comorbidity between substance use disorders and psychiatric conditions. Addiction 2006 Sep;101 Suppl 1:76-88.

37 Sewell RA, Poling J, Sofuoglu M. The effect of cannabis compared with alcohol on driving. Am J Addict. 2009 May;18(3):185-93.

38 Perra S, Pillolla G, Luchicchi A, Pistis M. Alcohol inhibits spontaneous activity of basolateral amygdala projection neurons in the rat: involvement of the endocannabinoid system. Alcohol ClinExp Res. 2008 Mar;32(3):443-9.

39 Kuhns JB, Wilson DB, Maguire ER, Ainsworth SA, Clodfelter TA. A meta analysis of marijuana, cocaine and opiate toxicology study findings among homicide victims. Addiction. $2009 \mathrm{Jul} ; 104(7): 1122-31$.

40 Chiang WK. Amphetamines. In: Nelson L, Lewin N, Howland MA, Hoffman R, Goldfrank L, Flomenbaum N, editors. Goldfrank'sToxicologic Emergencies. 9thed. New York: McGraw Hill; 2010.

41 Song BJ, Moon KH, Upreti VV, Eddington ND, Lee IJ. Mechanisms of MDMA (ecstasy)-induced oxidative stress, mitochondrial dysfunction, and organ damage. Curr Pharm Biotechnol. 2010 Aug;1 1(5):434-43.

42 Fiaschi AI, Cerretani D. Causes and effects of cellular oxidative stress as a resul of MDMA abuse. CurrPharmBiotechnol. 2010 Aug;11(5):444-52.

43 Moreno S. Lesiones de accidentes de transito Colombia 2010. Forensis $2011 ; 12(1): 254-94$

44 Fondo de Prevencion vial. Que el alcohol no afecta la conducción?. Bogotá: Fondo de Prevencion Vial; 2011.

45 Heng K, Hargarten S, Layde P, Craven A, Zhu S. Moderate alcohol intake and motor vehicle crashes: the conflict between health advantage and at-risk use. Alcohol Alcohol. $2006 \mathrm{Jul} ; 41(4): 451-4$.

46 Mockus A. Cultura ciudadana, programa contra la violencia en Santa Fe de Bogota, Colombia 1995 - 1997:estudio técnico. Washington: BID; 2001

47 Colombia. Senado.Resolución 000414 de 2002. CódigoNacional de Transito Terrestre

48 Odero W,ZwiAB. Alcohol-related traffic injuries and fatalities in developing coutries: A critical review of literature[monografía en Intemet]. London: London School of Hygiene and Tropical Medicine. Department of Public Health.Health Policy Unit; 1995 [citado 23 Mar 2012].Disponible en: http://www.druglibrary. org/schaffer/misc/driving/s26pl.htm

49 Odero W, Gamer P, Zwi A. Road traffic injuries in developing countries: a comprehensive review of epidemiological studies. Trop Med Int Health. 1997 May;2(5):445-60.

50 Garg N, Hyder AA. Exploring the relationship between development and road traffic injuries: a case study from India. Eur J Public Health. 2006 Oct;16(5):48791.

51 Hasin DS, Stinson FS, Ogbum E, Grant BF. Prevalence, correlates, disability, and comorbidity of DSM-IV alcohol abuse and dependence in the United States: results from the National Epidemiologic Survey on Alcohol and Related Conditions. Arch Gen Psychiatry. 2007 Jul;64(7):830-42.
52 Harwood HJ, Fountain D, Fountain G. Economic cost of alcohol and drug abuse in the United States, 1992: a report. Addiction. 1999 May;94(5):631-5.

53 Rehm J, Gnam W, Popova S, Baliunas D, Brochu S, Fischer B, et al. The costs of alcohol, illegal drugs, and tobacco in Canada, 2002. J Stud Alcohol Drugs. 2007 Nov;68(6):886-95

54 Walsh JM, Flegel R, Cangianelli LA, Atkins R, Soderstrom CA, Kems TJ. Epidemiology of alcohol and other drug use among motor vehicle crash victims admitted to a trauma center. Traffic Inj Prev. 2004 Sep;5(3):254-60.

55 National Highway Traffic Safety Administration. Drug Involvement of Fatally Injured Drivers[monografía en Internet].Washington: NHTSA; 2010.[citado 23 Mar 2012]. Disponible en: http://www-nrd.nhtsa.dot.gov/Pubs/811415.pdf

56 Goulle JP, Verstraete A, Boulu R, Costentin J, Foucher JP, Raes E, et al. [Illicit drugs, medications and traffic accidents]. Ann Pharm Fr. 2008 Aug;66(4):196205.

57 Cimbura G, Lucas DM, Bennett RC, Warren RA, Simpson HM. Incidence and toxicological aspects of drugs detected in 484 fatally injured drivers and pedestrians in Ontario. J Forensic Sci. 1982 Oct;27(4):855-67.

58 Mercer GW, Jeffery WK. Alcohol, drugs, and impairment in fatal traffic accidents in British Columbia. Accid Anal Prev. 1995 Jun;27(3):335-43.

59 Logan BK, Schwilke EW. Drug and alcohol use in fatally injured drivers in Washington State. J Forensic Sci. 1996 May;41(3):505-10.

60 Mason AP, McBay AJ. Ethanol, marijuana, and other drug use in 600 drivers killed in single-vehicle crashes in North Carolina, 1978-1981. J Forensic Sci. 1984 Oct;29(4):987-1026.

61 Soderstrom CA, Trifillis AL, Shankar BS, Clark WE, Cowley RA. Marijuana and alcohol use among 1023 trauma patients. A prospective study. Arch Surg. 1988 Jun;123(6):733-7.

62 Gerchow J. ["Other intoxicating drugs" in traffic law]. Blutalkohol. 1987 Jul;24(4):233-42.

63 Daldrup T, Reudenbach G, Kimm K. [Cannabis and alcohol in traffic]. Blutalkohol. 1987 Mar;24(2):144-56.

64 Drummer OH, Gerostamoulos J, Batziris H, Chu M, Caplehom JR, Robertson MD, et al. The incidence of drugs in drivers killed in Australian road traffic crashes. Forensic Scilnt 2003 Jul 8;134(2-3):154-62.

65 McLean S, Parsons RS, Chesterman RB, Dineen R, Johnson MG, Davies NW Drugs, alcohol and road accidents in Tasmania. Med J Aust 1987 Jul 6;147(1):6 11

66 Ramaekers JG, Berghaus G, van LM, Drummer OH. Dose related risk of moto vehicle crashes after cannabis use. Drug Alcohol Depend. 2004 Feb 7;73(2):10919.

67 Oladehinde MK, Adegbehingbe BO, Adeoye AO, Onakoya AO. Central nervous system stimulants: effect on visual functions and occurrence of road traffic accidents. Ann ItalChir. 2009 Jan;80(1):43-8.

68 Carmen del RM, Alvarez FJ. Presence of illegal drugs in drivers involved in fata road traffic accidents in Spain. Drug Alcohol Depend. 2000 Jan 1;57(3):177-82.

69 Reis AD, Figlie NB, Laranjeira R. Prevalence of substance use among trauma patients treated in a Brazilian emergency room. RevBrasPsiquiatr. 2006 Sep;28(3):191-5.

70 Rodríguez J, Acosta N, Peñaloza R. Carga de enfermedad Colombia 2005: Resultados alcanzados. Documento técnico. Bogotá: Pontificia Universidad Javeriana, Cendex; 2008

71 Constantin A. Reglamento técnico forense para la determinación del estado de embriaguez aguda. Bogotá: Instituto Nacional de Medicina Legal y Ciencias Forenses; 2005. 